\title{
Functional recovery in primates with brachial plexus injury after spinal cord implantation of avulsed ventral roots
}

\author{
Thomas P Carlstedt, Rolf G Hallin, Karl G Hedström, Ingela A M Nilsson-Remahl
}

\begin{abstract}
Intraspinal replantation of avulsed spinal nerve roots as a surgical treatment for motor deficits after severe brachial plexus injury was investigated in primates. Under general anaesthesia hemilaminectomy was performed in cynomogulus monkeys (Macaca fascicularis). Ventral roots within the brachial plexus were then avulsed by traction and subsequently implanted into the venterolateral aspect of the spinal cord. No dysfunction in the long fibre tracts was seen following surgery. Postoperatively there was a flaccid paralysis of the arm on the lesioned side. Severe atrophy developed within 5-7 weeks in the muscles supplied by the avulsed roots and EMG revealed denervation activity. Two to three months after surgery there were EMG signs of reinnervation, which were shortly followed by evidence of clinical recovery. A gradual improvement in the function of the affected arm occurred and the animals' motor behaviour normalised. One year after surgery there was a full range of motion in the arm, but the EMG activity in the reinnervated muscles at maximal force was reduced. Tracing of regenerated motor neurons with horseradish peroxidase (HRP) injected into the biceps muscle revealed retrogradely labelled motor neurons confined to the ipsilateral ventral horn. It was concluded that intraspinal replantation of avulsed ventral roots in primates significantly promotes motor recovery in the muscles supplied by the lesioned spinal cord segments.
\end{abstract}

(F Neurol Neurosurg Psychiatry 1993;56:649-654)

Nerve regeneration after ventral root avulsion and implantation into the spinal cord was demonstrated in a series of experiments in rats and cats. ${ }^{1-8}$ The reported results of neural regrowth may have clinical implications, but primate studies must be performed before the procedure can be used as a clinical routine in patients.

The aim of the present study was to investigate whether regeneration after ventral root avulsion and implantation also occurs in primates. The prime object was to elucidate a specific clinical situation; the root avulsion injuries that occur following a traumatic brachial plexus lesion. Thus primates were subjected to brachial plexus lesion with ventral root avulsions which were followed by implantation of the roots into the spinal cord. The outcome of the surgical procedure was evaluated clinically and electrophysiologically by performing EMG on the muscles supplied by the lesioned nerves. In addition, we report neuroanatomical findings which indicate neural regrowth. A significant clinical recovery occurred in the experimental animals subjected to an active therapeutic approach. Some aspects of the present results were briefly discussed elsewhere. ${ }^{9-11}$

\section{Material and methods}

In nine cynomogulus monkeys (Macaca fascicularis) a cervical hemilaminectomy was performed under general anaesthesia (Ketalar $0,1 \mathrm{~g} / \mathrm{kg} \mathrm{im}$ ) deep enough to abolish the corneal reflex. The dura was opened to expose the left lower cervical roots. Ventral roots were avulsed from their attachment with the spinal cord by traction with a fine hook (fig 1). In five monkeys the ventral roots $\mathrm{C} 5, \mathrm{C} 6$ and $\mathrm{C} 7$, and in two animals the C5Th1 ventral roots were avulsed. Immediately following injury the roots were inserted into the spinal cord through its venterolateral aspect, ventral to the denticulata ligament. The avulsed roots were long enough to allow implantation into the spinal cord without nerve grafts. The position of the tips of the roots, just below the pial surface of the spinal cord, was retained with tissue glue (Tisseel ${ }^{\circledR}$ ) or $9 / 0$ stay sutures (Ethilon ${ }^{\Phi}$ ). In two additional animals the avulsed roots were not implanted but left detached in the subdural space. The dural incision was closed with $6 / 0$ sutures and the wound closed in layers.

The behaviour of the animals in situations, such as, eating and climbing was followed regularly and videotaped every second week. Conventional EMG recordings were made with concentric needle electrodes (Medelec Ltd) and a Medelec MS 91 recording apparatus. These studies were mainly conducted in muscles supplied by the lesioned root(s) but in a few animals EMG was also performed in corresponding muscles of the normal limb. The biceps muscles were investigated bilaterally. To carry out the EMG recordings the animals were sedated with Ketalar. A dose of $0.05 \mathrm{~g} / \mathrm{kg}$ administered intramuscularly once or twice was adequate to keep the animals calm but awake in a recumbent position. The occurrence of denervation activity was studied when the animal was relaxed. Voluntary 


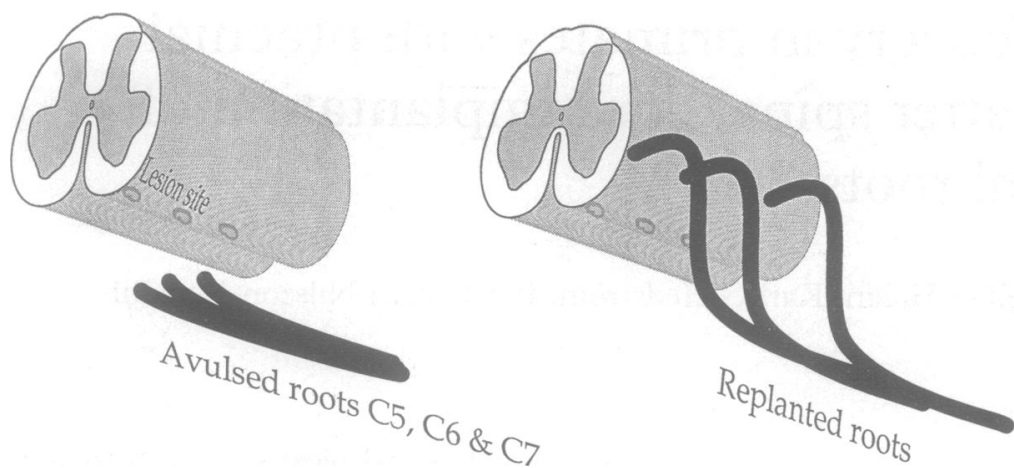

Figure 1 Schematic illustration of the method of ventral root avulsion and subsequent replantation of the avulsed roots.

contraction of the biceps muscle was achieved by pulling the hand of the monkey away from the body and simultaneously alerting him with somatosensory stimulation, such as pinching the hand or rubbing the sternum.

One year after surgery the animals were subjected to tracing studies by injecting a solution of horseradish peroxidase, HRP, (50 $\mathrm{mg} / \mathrm{ml}$ Ringer solution) into the biceps muscle. Two days later the animals were sacrificed, under general anaesthesia, by vascular perfusion with $5 \%$ glutaraldehyde in a 300 mOsm phosphate buffer through a cannula inserted into the left ventricle of the heart. The cervical spinal cord was dissected out. The C6 spinal cord segment was cut into $50 \mu \mathrm{m}$ thick transverse sections in a cryostat. The sections were incubated with tetramethylbenzidine and examined in the light microscope. The transverse distribution of the neurons in the ventral horn was plotted.

The surgical, behavioural, and electrophysiological procedures used were all approved by the ethical committee of Karolinska Institute.

\section{Results}

CLINICAL APPEARANCE

Immediately postoperatively there was a flaccid paralysis in the shoulder and elbow. If all roots in the plexus (C5-Th1) were avulsed, function in the hand was also abolished. None of the animals exhibited any signs indicating damage to long fibre tracts, such as spasticity or anaesthetic skin areas. The patellar and Achilles tendon reflexes were symmetrical and normal and the Babinski sign was absent bilaterally. The first three months after surgery the monkeys did not use the lesioned arm in activities such as climbing or eating (fig 2A, 3A, B). The arm hung along the thorax wall (fig $2 \mathrm{~A}, 3 \mathrm{~A}, \mathrm{~B}$ ). The first signs of clinical restitution were apparent more than three months after surgery. The monkey used the lesioned arm to hold objects, such as fruits, together with the unoperated arm. Occasionally the animal raised food to its mouth, but the most common eating behaviour at this time was to bend forward and eat with the fruit on a support, like a shelf or the floor of the cage (fig 3A). A gradual improvement in the function of the affected arm occurred. Five months after surgery the animals lifted food regularly in a two handed grip to the mouth. Without objects in the hand the lesioned arm could be flexed fully in the elbow. Support of the lesioned arm while climbing (fig 2B) occurred only occasionally. After six months, there was almost full range of motion in the shoulder and elbow. The monkeys regularly used the lesioned arm for
Figure 2 Climbing behaviour: A): (lefi) One month after $\mathrm{C} 5-\mathrm{C} 7$ ventral root avulsion and implantation on the left side. The left arm is flail (arrow) and not used in climbing. Note that the monkey compensates for the useless left arm, by supporting itself with a foot (double arrow). B) (right) The same monkey, six months after surgery. The previously paralysed left arm (arrow) is used during climbing.

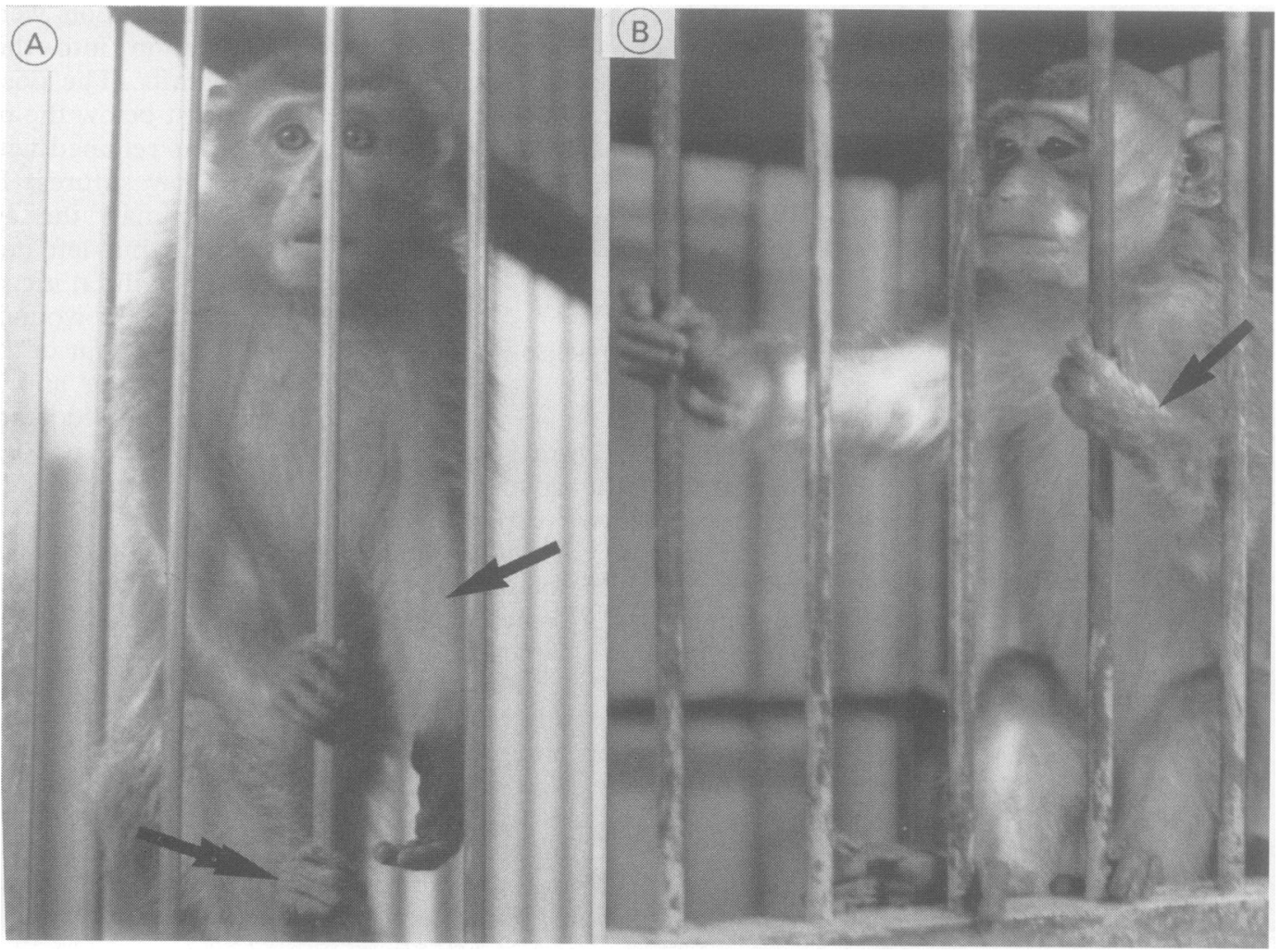



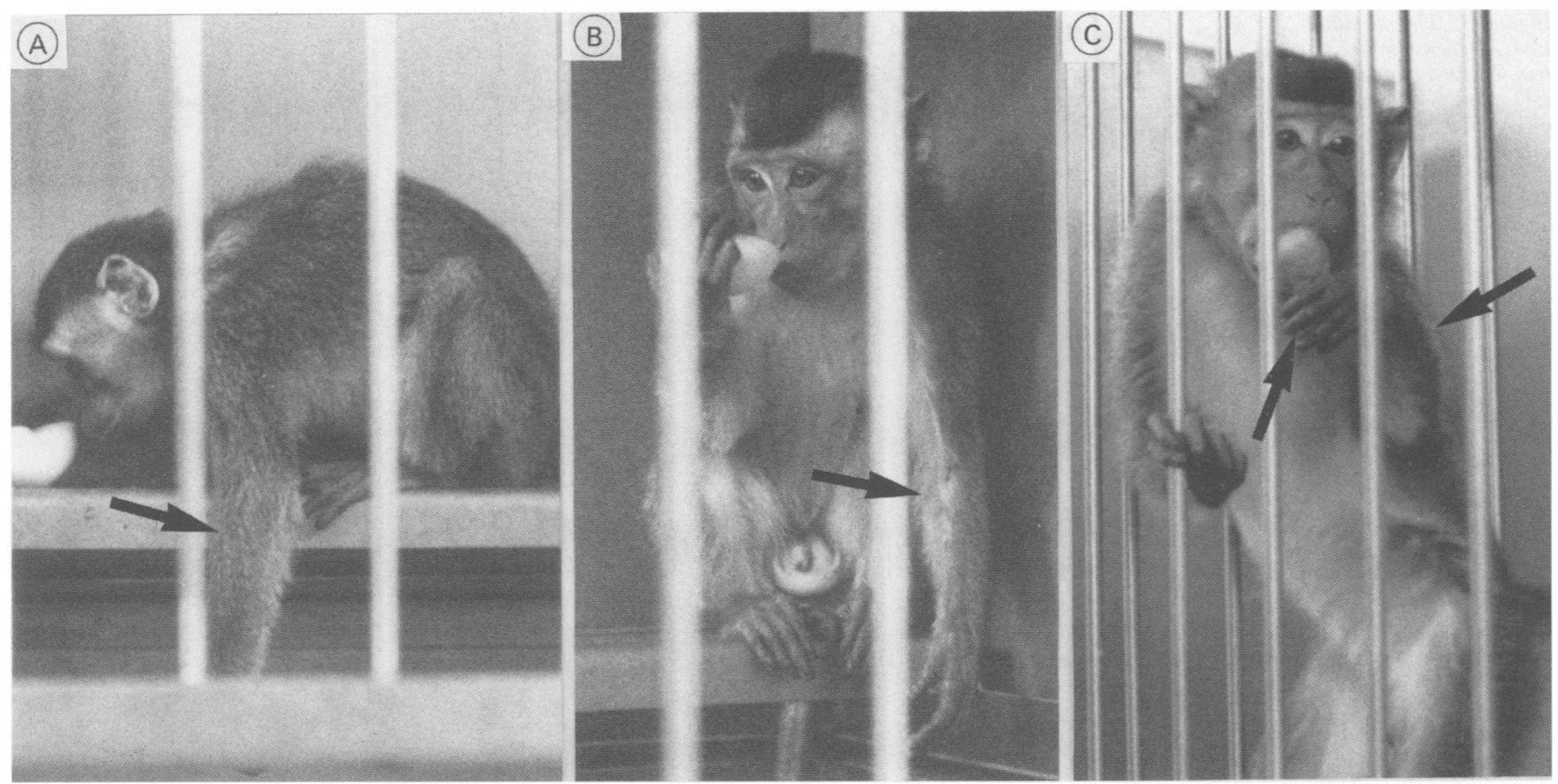

Figure 3 Eating behaviour: A (left) B: (Centre) One month after C5-C7 ventral root avulsion and implantation on the left side. The left arm (arrow) is not used during eating. The monkey bends over to eat from a support $(A)$ or uses only the right arm $(B)$. $C$ (right): The same monkey after six months. The unoperated right arm supports the animal on the cage while the previously paralysed left arm (arrow) is used to lift food to the mouth. Note the volar flexion of the left hand caused by insufficient recovery of forearm extensors.

support while climbing and the eating behaviour had changed. They now lifted fruits to their mouth (fig 3C), instead of bending forward to eat from a shelf or the floor of the cage. However, they generally preferred to use the unoperated arm (fig 3B). This clinical appearence was consistent in the three animals who had sustained a partial lesion of the brachial plexus (C5-C7). They showed an almost normal motor behaviour of the arm after one year. Animals with a total lesion (C5-Th1) did not recover full hand function even after long survival times postoperatively $(>1$ year). Due to impaired hand function these animals disregarded the lesioned arm and developed joint contractures. In animals who had sustained avulsion injuries without subsequent implantation of the avulsed roots, clinical signs of restitution were not seen.

There was a strikingly low skin temperature in the hand of the lesioned side compared with the control side. The reduced hand temperature was most pronounced in the animals with total root avulsions of the plexus. The difference in hand temperature between the lesioned and control side sometimes amounted to more than $3^{\circ} \mathrm{C}$ (ambient temperature about $35^{\circ} \mathrm{C}$ ).

MOTOR FUNCTION

Severe atrophy developed within 5-7 weeks in the muscles supplied by the avulsed nerve roots. Following total root avulsion and root implantation there was electromyographic silence in the affected muscles for 3-5 weeks when denervation activity was generally present. Sometimes motor unit activity under voluntary control was still present after the avulsion trauma in the supposedly totally denervated muscles. Whenever such electromyographic signs of remaining innervation, indicating incomplete denervation, were demonstrated immediately after operation, the animal was discarded from the study. (Two of the seven experimental animals). At the peak of atrophy there was abundant denervation activity in the biceps muscle (fig 4), which on the lesioned side was reduced to a thin streak of elastic tissue, contrasting with the bulky appearance of the muscle on the control side. Electromyographic signs of reinnervation preceded the clinical improvement which first appeared as local tiny muscle twitches after about three months. The reinnervation potentials were generally monophasic and of low amplitude and appeared most distinctly when the animal was semiconscious and recumbent in a relaxed position. In parallel with the clinical improvement of the animal, there was an increase in the amplitude of the optimally recorded EMG activity encountered in response to apparently maxi-
Figure 4 Abundant denervation activity was recorded in the biceps muscle on the injured side 3 to 5 weeks post surgery. Calibrations $20 \mu \mathrm{V}$ respectively $20 \mathrm{~ms}$ (vertical and horizontal bars).

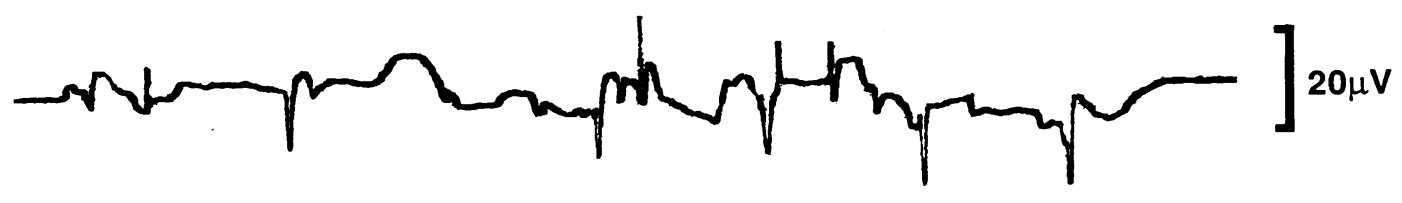


Figure 5 The maximal EMG response in the reinnervated biceps muscle (A) was $20-40 \%$ of that on the control side

(B) after up to 12 months. Calibrations $200 \mu V$ and $100 \mathrm{~ms}$ (vertical and horizontal bars) and $500 \mu \mathrm{V}$ and $100 \mathrm{~ms}$ in $\mathrm{A}$ and $B$ respectively.
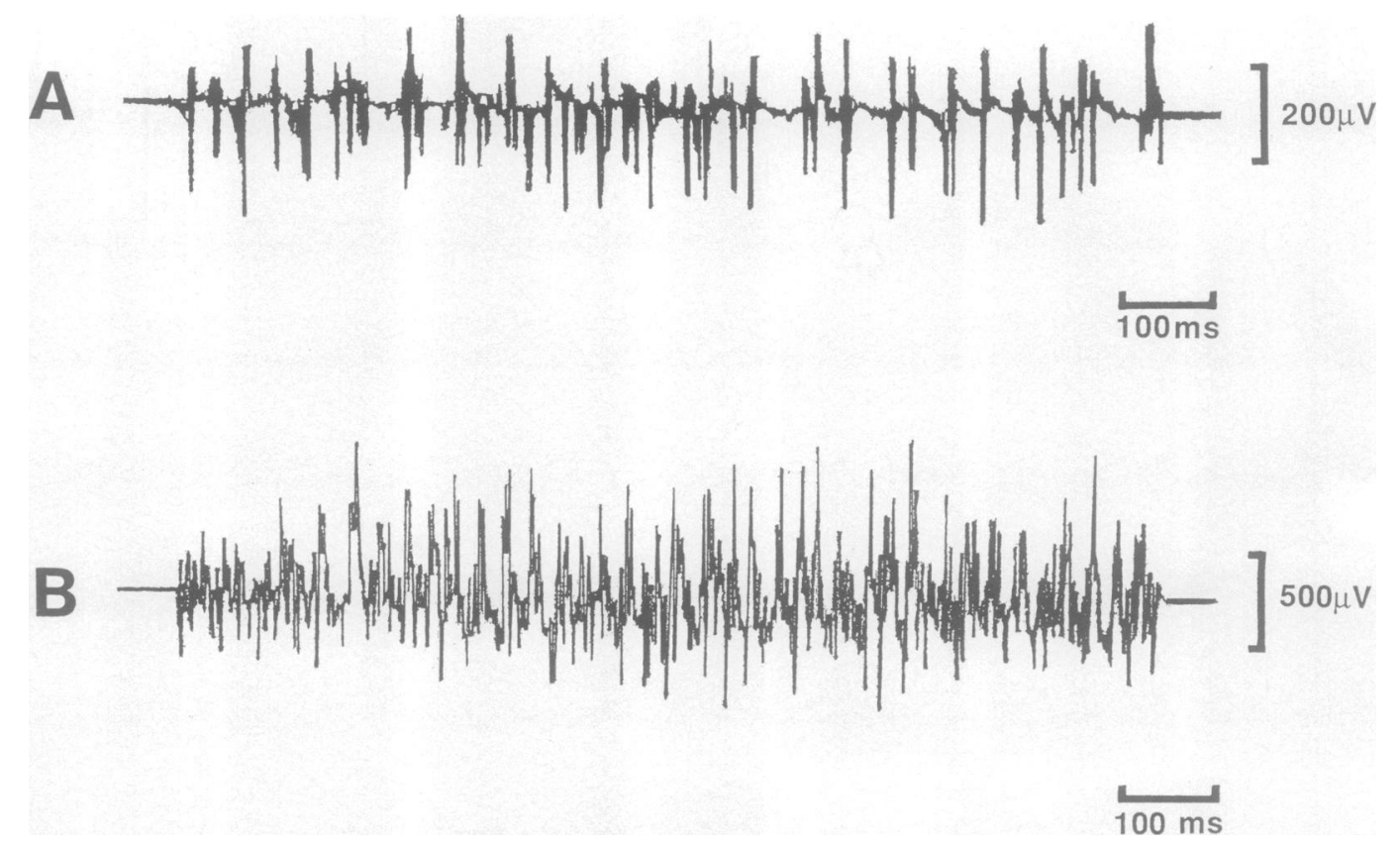

mal voluntary contraction. After long survival times the maximal EMG response in the reinnervated biceps muscle increased to about $20-40 \%$ of that on the control side (fig 5 ). In the best animal the maximal EMG response in the reinnervated biceps muscle was about $50 \%$ of that on the control side. In this case the bulky appearance of the muscle was striking and the circumference of the arm was $70-80 \%$ of that on the normal side.

\section{Morphology}

The tips of the implanted roots were located just deep to the spinal cord surface and close to, but not inside the ventral horn. HRP labelled cells were dispersed throughout the ipsilateral ventral horn (fig 6). The number of retrograde labelled HRP neurons in the C6 segment varied between 97 to 137 . The size of the neurons showed large variations with a mean diameter of the cell body ranging between $20 \mu \mathrm{m}$ and $80 \mu \mathrm{m}$ (mean $49 \mu \mathrm{m}$ ). In some cases axons of the labelled cells could be traced towards the implanted root (fig 7).

\section{Discussion}

Traction lesions of the brachial plexus in adult humans are usually the result of motorcycle accidents and in children due to complicated delivery. The brachial plexus injury is usually complex with both spinal nerve and spinal root ruptures together with avulsion of one or several roots from the spinal cord. ${ }^{12}$ Today spinal nerve ruptures can be managed according to routine surgical principles for peripheral nerve injuries with a functional outcome. ${ }^{13}$ The root avulsion injuries are not repaired, however, since they are regarded as lesions of the CNS. The population of neurons located in the spinal cord segment denervated by root avulsions therefore remain detached from their terminals in the periphery. For regeneration to occur in the avulsed roots, nerve transfer or neurotisation can be 
performed with foreign adjacent nerves like the accessory nerve or intercostal nerves. ${ }^{14} \mathrm{~A}$ considerably impaired motor function in the arms and the hand/s is, however, the usual outcome of these injuries. ${ }^{15}$

In attempts to find ways to manage the motor deficits following complex nerve root injuries, the outcome of experimental studies of ventral root avulsion and reimplantation in the spinal cord were encouraging. ${ }^{156}$ In rats and cats motor neurons in the lumbar spinal cord reinnervated ventral root implants after an avulsion of ventral roots at the spinal cord surface. ${ }^{16}$ Reinnervation of the implanted ventral root was achieved after an initial growth of new axons in the CNS. ${ }^{6}$ By the use of intracellular recording in the spinal cord and staining with horseradish peroxidase it was found that alpha and probably also gamma motor neurons were able to reinnervate ventral root implants. ${ }^{6}$ The labelled neurons were excited or inhibited by impulses in afferent fibres and their contribution to elicited reflex activity was normal, so that muscle twitch responses were induced by electrical stimulation of the implanted root. These previous experiments unequivocally demonstrated axonal regrowth from the motor neuron pool through CNS tissue in the spinal cord to the implanted ventral root/s. Other routes of regrowth seemed unlikely in these trials since the experimental procedure involved extensive excision of adjacent roots and intracellular labelling of the regenerated neurons. ${ }^{6}$ As expected, the present morphological and tracing studies in monkeys also demonstrated re-established anatomical links between the motor neuron pool, the implanted ventral roots and the muscles. The population of HRP-labelled motor neurons was not confined to the anatomical site in the ventral horn normally supplying the proximal part of the limb. Instead, the labelled cells were diffusely localised in the ventral horn indicating that the regeneration was unspecific involving different motor neuron pools irrespective of their previous destination.

In this study in primates other issues of possible clinical significance were primarily addressed. Thus all avulsed roots were implanted and adjacent roots were left intact. Our findings demonstrate that such a surgical procedure executed after avulsion injuries of the brachial plexus can promote motor recovery in primates. A similar effect was not seen in animals that did not receive an implant. It is suggested that the functional outcome of the surgery to a large part was dependent upon nerve regeneration through the spinal cord into the implanted nerve root(s). However, regeneration from other sources might have contributed to the final clinical outcome of the procedure since precautions to eliminate regeneration through, for example, extraspinal routes were not taken in these studies. Regrowth from the avulsion site along the pia mater to the lesioned roots that is, a peripheral nervous system type of regeneration, might have occurred. Extensive sprouting and regeneration of motor neuron axons invading the pia mater was previously described after radicular injuries. ${ }^{16-18}$ In a separate study where the reinnervation process was evaluated in detail with EMG we obtained reliable evidence that suggested extraspinal regeneration in the present paradigm (unpublished material). Alternative pathways for elongating motor axons after ventral root avulsion was recently confirmed in the rat. ${ }^{8}$ The present experiments demonstrate that the surgical method to implant the avulsed root superficially into the spinal cord induces a functional recovery, probably based on regrowth through both the CNS and the PNS. Neurophysiological signs of denervation in the biceps muscle of operated animals with ventral root implantation was followed by evidence of reinnervation as shown by repeated EMG investigations. Such a sequence of events was not documented in animals without implantation after avulsion. Reinnervation potentials in the EMG preceded the clinical signs of improvements, like muscle contractions, which occurred about three months after surgery. As the distance from the spinal cord to the biceps muscle is about $60 \mathrm{~mm}$, axonal regeneration at $1 \mathrm{~mm}$ per day would fit with the time interval between surgery and clinical signs or restitution. ${ }^{12}$

An implantation procedure was previously used clinically to restore function in avulsed dorsal roots. ${ }^{19}$ Surgical trauma to the exposed intact spinal cord might induce a functional loss caudal to the lesion. In our experiments the avulsed roots were introduced into the cord just ventral to the denticulata ligament. A lesion at this location is not likely to affect the long descending motor tracts but could damage fibres of the ascending spinothalamic tract. However, there were no clinical signs indicative of injury in the long fibre tracts such as spasticity, motor deficits or anaesthesia in the lower body parts in any of the animals. Thus the implantation procedure as such does not seem to involve any major disadvantages.

We concluded that, as in peripheral nerve lesions, ${ }^{2021}$ an active therapeutic approach can promote motor regeneration even after severe brachial plexus injuries.

This study was supported by the Swedish Medical Research Council (project 7127), Cancer och Trafikskadades Riksförbund, Folksam Insurance Company, Clas Groschinsky's Minnesfond, the Karolinska Institute, and Torsten och Ragnar Söderberg's foundations. The excellent technical assistance of Ms Anita Bergstrand and the expert
secretarial aid of Kerstin Morén is gratefully acknowledged.

1 Carlstedt $T$, Lindå $H$, Cullheim $S$, Risling $M$. Reinnervation of hindlimb muscles after ventral root Reinnervation of hindlimb muscles after ventral root
avulsion and implantation in the lumbar spinal cord of avulsion and implantation in the lumbar spinal cord

the adult rat. Acta Physiol Scand 1986;128:645-6.
Carlstedt T, Cullheim S, Risling M, Ulfhake B. Mammalian root-spinal cord regeneration. In DM Gash and JR Sladek, Jr (Eds.) Transplantation into the Mammalian CNS, Progress in brain research: vol. 78, Amsterdam: Elsevier, 1988:225-9.

3 Carlstedt T, Cullheim S, Risling $M$, Ulfhake U. Nerve fibre regeneration across the PNS-CNS interface at the root-spinal cord junction. Brain Res Bull 1989;22: 93-102.

4 Carlstedt $T$, Risling $M$, Lindå $H$, Cullheim S, Ulfhake B Sjögren AM. Regeneration after spinal nerve root injury. Restor Neurol Neurosci 1990;1:289-95. 
5 Horvat J-C, Pécot-Dechavassine M, Mira J. Reinnervation fonctionelle d'un muscle squelettique du Rat adulte au moyene d ún greffon de nerf périphérique introduit dans la moelle épinère par voi dorsal C.r. hebd. Séanc. Acad Sci Paris 1987;304:143-48.

6 Cullheim S, Carlstedt T, Lindå H, Risling M, Ulfhake B. Motoneurons reinnervate skeletal muscles after ventral root implantation into the spinal cord of the cat. root implantation into

7 Hoffmann CFE, Thomeer RTWM, Marani E. Ventral root avulsion of the cat spinal cord at the brachial plexus root avulsion of the cat spinal cord at the brachial plexus level (Cervical $1990 ; 28: 418-29$.

8 Smith KJ, Kodama RT. Reinnervation of denervated skeletal muscle by central neuron regenerating via ventral roots implanted into the spinal cord. Brain Res 1991;551:221-9.

9 Carlstedt T, Aldskogius H, Hallin RG, Nilsson-Remahl I. Novel surgical strategies to correct neural deficits following experimental spinal nerve root lesions. Brain Research Bulletin 1992;30:405-13.

10 Carlstedt T, Hallin, RG, Nilsson-Remahl, I. Absence of Carlstedt $T$, restitution after implantation of avulsed ventral roots in primate brachial plexus injury The fournal of Reconstructive Microsurgery 1993 (In press)

11 Carlstedt T, Hallin RG, Nilsson-Remahl J. Functional motor recovery after extensive root avulsions followed by ventral root implantation in primates. Electroenceph. Clin Neurophysiol. 1992;83.
12 Sunderland, S. Nerve and nerve injuries. Edinburgh: Churchill-Livingstone, 1978

13 Alnot J.Y. Paralysie traumatique du plexus brachial chez I'adult. Rev Chir Ortop 1977;63:17-125.

14 Narakas AO. Thought on neurotization or nerve transfers in irreparable nerve lesions. Clin Plast Surg 1984;11: 153-60.

15 Alnot JY. Paralysie traumatique du plexus brachial de I'adulte. Encycl. Medico-Chir (Paris) 1983;9:1-15.

16 Ramón y Cajal S. Degeneration and regeneration of the nervous system, New York: Hafner Press, Macmillan, 1928.

17 Risling M, Hildebrand C, Cullheim S. Invasion of the L7 ventral root and spinal pia mater by new axons after sciventral root and spinal pia mater by new axons after sci83:84-97.

18 Risling $M$, Carlstedt $T$, Lindå $H$, Cullheim $S$. The pia mater-a conduit for regenerating axons after ventral root replantation. Rest Neurol Neurosci 1991;3:157-60.

19 Jamiesson AM, Eames RA. Reimplantation of avulsed brachial plexus roots: an experimental study in dogs. In f Microsurg 1980;2:75-80.

20 Hallin RG, Wiesenfeld Z, Lindblom $U$. Neurophysiological studies on patients with sutured median nerves: faulty sensory localization after nerve regeneration and its physiological correlates. Exp Neurol 1981;73:90-106.

21 Hallin RG, Wiesenfeld $Z$, Lugnegård $H$. Neurophysiological studies of peripheral nerve functions after physiological studies of peripheral nerve functions after Rehab Med 1981;3:187-92. 